\title{
Artifacts, Activities, Benefits and Blockers: Exploring Enterprise Architecture Practice in Depth
}

\author{
Sherah Kurnia \\ University of Melbourne \\ sherahk@unimelb.edu.au \\ Paul Taylor \\ DHHS Victoria \\ paul.taylor@dhhs.vic.gov.au
}

\author{
Svyatoslav Kotusev \\ HSE University \\ kotusev@kotusev.com \\ Graeme Shanks \\ University of Melbourne \\ gshanks@unimelb.edu.au
}

\author{
Rod Dilnutt \\ University of Melbourne \\ rpd@unimelb.edu.au \\ Simon Milton \\ University of Melbourne \\ milton.simon@gmail.com
}

\begin{abstract}
Enterprise architecture (EA) is a collection of artifacts describing an organization from an integrated business and IT perspective and intended to improve business and IT alignment. The purpose of this study is to identify benefits and blockers associated with specific EA-related activities and respective artifacts. Most existing studies discuss the benefits and problems of EA practice in general without relating them to specific activities constituting EA practice. This study is based on 18 interviews with architects and leverages the grounded theory approach. As a result of our analysis, we identify eight consistent activity areas constituting EA practice. Each activity area implies certain activities supported by some EA artifacts leading to specific benefits often impeded by some blockers. Our analysis indicates that EA practice includes many diverse activities usually, though not always, closely associated with specific types of EA artifacts. Moreover, benefits and blockers of EA practice are also very activity-specific.
\end{abstract}

\section{Introduction}

In the digital epoch achieving business and IT alignment still remains among the topmost concerns of IT executives. Enterprise architecture (EA) is a collection of special documents, typically called as artifacts, describing various aspects of an organization from an integrated business and IT perspective $[1,2]$. EA facilitates communication between business and IT stakeholders and helps improve business and IT alignment.

Various EA artifacts used in organizations can be very diverse in nature and range from executive-level architecture principles [3] and core diagrams [4] to rather detailed and technical project-start architectures [5]. These EA artifacts have different usage scenarios in organizations ranging from guiding IT investments [6] to ensuring compliance of separate IT projects with an organization-wide architecture [7]. EA practice, as an organizational activity that implies using EA artifacts, may also include a variety of diverse actions permeating the whole organization from top-level corporate strategic management [8] to mid-level IT portfolio management [9] to separate system development processes on the ground [10].

Previous studies identified a number of benefits and problems associated with "EA management" [11, 12], "EA programs" and "EA projects" $[13,14]$ or even simply "enterprise architecture" $[15,16]$. However, none of these studies distinguished any concrete activities constituting EA practice and analyzed the benefits and problems specifically in relation to these particular activities.

In order to address this gap, this study explores EA practices at a more detailed level and identifies the benefits and blockers associated with specific EArelated activities and EA artifacts. Put it simply, this study aims to "connect" various EA artifacts and activities with corresponding benefits and blockers. The research question of this study can be formulated as follows: "How are different benefits and blockers associated with specific EA-related activities and their respective EA artifacts?"

This paper is structured as follows. Section 2 discusses the related works on EA artifacts and EA practice outlining its activities, benefits and blockers. Section 3 describes our research method, data collection and analysis procedures. Section 4 describes eight identified activity areas of EA practice. Section 5 discusses our findings. Finally, Section 6 concludes the paper. 


\section{Related works}

\subsection{Enterprise architecture and its artifacts}

EA consists of multiple separate components typically called as EA artifacts [1,2]. An EA artifact is a descriptive document providing a certain view of an organization from the perspective of its business and IT $[1,2,17]$. Various EA artifacts used in organizations as part of EA practices can be very diverse in nature. For instance, popular EA artifacts include architecture principles [3], standards [18], core diagrams [4], business capability models or maps [19], enterprise data models [20], project-start architectures [5] and many other different types of EA artifacts [1, 21].

Various EA artifacts have different use cases and usage scenarios in organizations $[1,2]$. For example, business capability models can be used for improving strategic business and IT alignment [22] or to support acquisitions of other companies [23]. More formal technical EA diagrams can be used to analyze availability of IT systems [24] or service response times [25]. Architecture principles can be used to guide IT investments [6], whereas project-start architectures can be used to ensure compliance of separate IT projects with an organization-wide architecture [7]. Therefore, both EA artifacts themselves and their use cases can be very different.

\subsection{Enterprise architecture practice and its activities}

EA practice is an organizational effort that implies using EA artifacts for facilitating IT-related decisionmaking and improving business and IT alignment [26]. EA practices in organizations imply a variety of diverse activities integrated with other organizational activities [4, 27, 28]. For example, at the top executive level EA practices can be integrated with corporate strategic management [8], strategic planning [29] and business model management [30]. At the middle management level EA practices can be intertwined with risk management [31], information management [32] and change management [33]. At the portfolio level EA practices can be integrated with IT portfolio management [9] and investment selection processes $[10,34]$. Finally, at the project implementation level EA practices may interact with system development processes [10, 34]. Hence, EA practices in organizations imply a broad spectrum of disparate activities.

\subsection{Benefits of enterprise architecture practice}

Benefits of EA practice is among the most extensively studied topics in the available EA literature and numerous studies have been conducted to analyze them [35]. For example, Bradley et al. [15] demonstrate that business and IT alignment, the overall value of IT and the quality of risk management all positively correlate with the maturity of EA. Schmidt and Buxmann [11] prove statistically that EA practices lead to increased IT efficiency and IT flexibility, which itself leads to greater IT connectivity, compatibility and modularity. Valorinta [36] demonstrates that the use of EA improves business and IT alignment. Bradley et al. [37] show that business and IT alignment, operational IT effectiveness and enterprise agility are positively related to the maturity of EA. Alaeddini and Salekfard [14] prove that the execution of EA projects leads to business and IT alignment through the six relevant aspects: communications, value measurements, governance, partnership, skills and scope. Hence, the use of EA leads to various benefits for organizations.

\subsection{Blockers of enterprise architecture practice}

EA practices are traditionally associated with numerous problems, challenges and pitfalls, which are widely discussed in the available EA literature [35]. For example, case study-based investigations of EArelated problems and blockers have been carried out in the United States [13], continental Europe [12], Norway [38], Finland [39] and Vietnam [16]. Extensive interview-based studies of EA-related obstacles have been conducted by European researchers [40] and less extensive ones by their South African colleagues [41]. Hauder et al. [42] organized a global survey of EA practitioners to examine the challenges related to EA practices. These studies identified various blockers of EA initiatives ranging from the scarcity of experienced architects $[42,43]$ and the absence of sufficient resources to develop complete EA documentation $[12,39]$ to inadequate communication [43] and organizational politics [16, 41]. Therefore, blockers of EA practices in organizations may be very diverse.

\section{Research method}

Since our research question addresses a previously unexplored area of the EA discipline, this study is considered as an early exploratory investigation. Although some empirically validated lists of EA 
artifacts that can be used as a basis for research purposes have been developed [1], no such lists are available for EA-related activities, benefits and blockers. Therefore, our research question is qualitative in nature and can hardly be answered with any quantitative research methods, e.g. structured mail questionnaires or surveys.

\subsection{Data collection}

Our research question is company-agnostic and does not focus on separate organizations or specific industries, but rather relates to EA practice in general. In order to exclude potential organization-specific bias in our findings, we avoided in-depth case studies of a limited number of companies. Instead, we achieved a broad coverage of EA practice and interviewed multiple experienced EA practitioners representing different companies and industry sectors. We selected a semi-structured interview survey as the most appropriate research method for our study [44]. In particular, we conducted 18 one-hour interviews in total with practicing architects and architecture managers having at least five years of active industry experience in EA. All interviews were guided by a standardized interview protocol covering four key topics of interest: artifacts, activities, benefits and blockers. Table 1 provides a brief profile of our research participants.

\begin{tabular}{|l|l|l|l|l|l|l|l|}
\hline$\#$ & Title & Industry & Experience & $\#$ & Title & Industry & Experience \\
\hline 1 & Enterprise Architect & Retail & $\sim 7$ years & 10 & Enterprise Architect & Government & $\sim 8$ years \\
\hline 2 & Architecture Manager & Diversified & $\sim 13$ years & 11 & Architecture Manager & Retail & $\sim 10$ years \\
\hline 3 & Enterprise Architect & Bank & $\sim 8$ years & 12 & Head of Applications & Retail & $\sim 10$ years \\
\hline 4 & Lead Architect & Telecom & $\sim 10$ years & 13 & Chief Architect & Healthcare & $\sim 7$ years \\
\hline 5 & Chief Architect & Finance & $\sim 15$ years & 14 & Architecture Manager & Resources & $\sim 11$ years \\
\hline 6 & Lead Architect & Healthcare & $\sim 6$ years & 15 & Information Architect & Government & $\sim 5$ years \\
\hline 7 & Architecture Manager & Retail & $\sim 12$ years & 16 & Architecture Manager & Finance & $\sim 12$ years \\
\hline 8 & Enterprise Architect & Steel & $\sim 10$ years & 17 & Enterprise Architect & Public services & $\sim 6$ years \\
\hline 9 & Head of Architecture & Bank & $\sim 20$ years & 18 & Chief Architect & Telecom & $\sim 17$ years \\
\hline
\end{tabular}

Table 1. Profile of research participants interviewed in our study

\subsection{Data analysis}

Due to the exploratory, qualitative and inductive nature of this study, all the collected interview data has been analyzed with the grounded theory method [45], which can be considered as the most appropriate data analysis method when no established theories exist in the relevant subject area. Hence, for data analysis we followed the three essential steps of the grounded theory method: open coding, axial coding and selective coding [45].

The first step, open coding, involved reading the transcribed interviews line-by-line and identifying significant concepts relevant to our research question, e.g. different types of EA artifacts, activities, benefits and blockers. The second step, axial coding, implied rereading the transcribed interviews and establishing the relationship between various concepts identified previously during the open coding step. The final step, selective coding, included selecting EA-related activities as the core category and unifying all the previously established concepts, categories and relationships between them around this category into eight consistent higher-order activity areas answering our research question.

\section{Eight activity areas of enterprise architecture practice}

Our grounded theory analysis of the collected interview data identified eight consistent activity areas mentioned in some or the other form by multiple EA practitioners. These activity areas are organized around respective actions of architects and reflect different aspects of their work in organizations. Each of these activity areas implies certain activities supported by some EA artifacts (though some activities cannot be clearly associated with any particular types of EA artifacts) leading to specific organizational benefits that are often impeded by some blockers. Figure 1 depicts these eight activity areas including relevant artifacts, activities, benefits and blockers. Brief descriptions of the eight identified activity areas are provided below. 


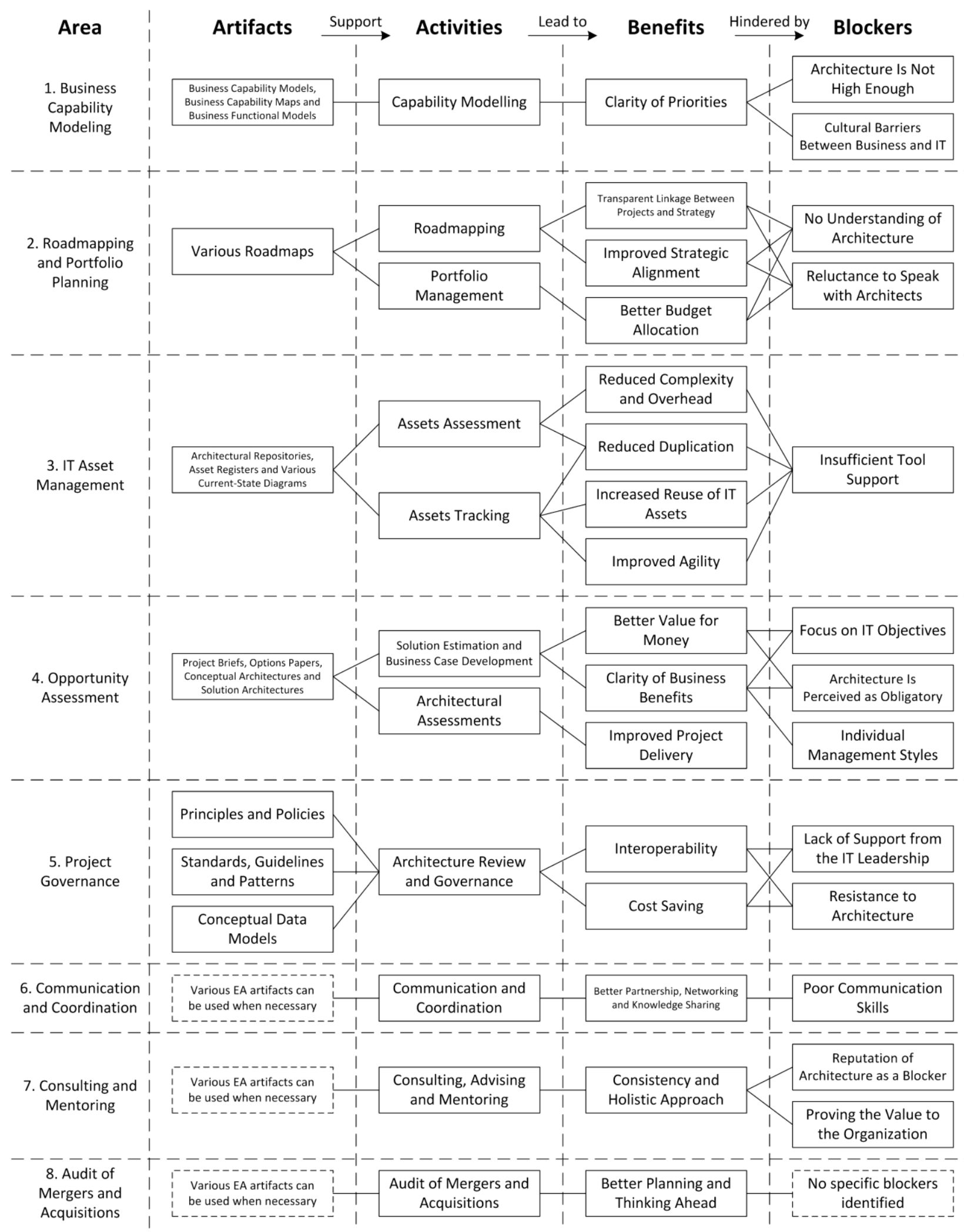

Figure 1. Eight major activity areas constituting enterprise architecture practice 


\subsection{Business capability modeling}

The business capability modeling activity area encompasses all activities of architects related to dealing with business capabilities. These activities often include identifying organizational business capabilities, assessing their relative maturity (often against external industry benchmarks), articulating necessary capability improvements and "heatmapping" respective capabilities to indicate future priorities for IT investments.

This activity area is normally supported by EA artifacts commonly known as business capability models, or less often business capability maps and highly similar business function models. These EA artifacts provide holistic one-page views of the whole organization from the perspective of its business capabilities or activities often also describing their underlying components, e.g. people, processes, information and systems.

All organizational benefits associated with this activity area can be broadly summarized to the clarity of priorities. Business capability modeling helps business executives and architects agree on the set of strategic business capabilities, discuss their priority and criticality to the organization, develop a shared understanding of their required maturity levels and propose some IT investment programs intended to uplift their maturity.

The first blocker associated with the business capability modeling activity area identified from the interviews is the situation when architecture is not positioned high enough in the organizational hierarchy to access senior business executives. The second common blocker related to this activity area is the general cultural alienation between business and IT sometimes described as "us and them" culture.

"You will have the commissioner of IT, then he will have a director underneath him and then enterprise architects will be one or two layers below. So, they are three or four levels below any serious decision making" (\#6)

\subsection{Roadmapping and portfolio planning}

The roadmapping and portfolio planning activity area encompasses all activities of architects related to defining future IT initiatives. These activities often include proposing new IT initiatives aligned to strategic business priorities, arranging these initiatives based on their importance, mutual dependence and deadlines, scheduling their execution at specific time intervals and finally shaping the IT investment portfolio based on the tactical priorities for the upcoming budgetary period, often for the next financial year.

This activity area is typically supported by various sorts and "flavors" of architecture roadmaps where all envisioned IT initiatives are depicted, e.g. investment roadmaps, application roadmaps and technology roadmaps. These roadmaps show all planned IT investments in different business units, areas or capabilities, their commencement and completion dates and in some cases also respective target states in terms of necessary information systems or capability maturity levels.

Typical organizational benefits associated with this activity area all relate to improved alignment, traceability and linkage between business strategy and IT initiatives. Roadmapping and portfolio planning helps business leaders and architects synchronize business and IT plans, develop mutually agreed delivery schedules for IT projects and allocate adequate financial, human and organizational resources required for their implementation. These activities also allow balancing strategic and tactical imperatives and achieving a more proactive business ownership of IT.

The first popular blocker associated with the roadmapping and portfolio planning activity area reported by the interviewees is the lack of understanding, if not ignorance, of architecture among business leaders that undermines productive communication. The second blocker related to this activity area is the reluctance of business managers to discuss their future plans and needs with architects, which also harmful for the quality of dialog between business and IT.

"The number one blocker [is that] I do not think there are many people in the organization who actually had any exposure to EA. [...] I have to convince these people that [roadmapping] is useful to them" (\#4)

\subsection{IT asset management}

The IT asset management activity area encompasses all activities of architects related to managing existing IT assets in the organization. First, these activities include all the actions necessary to keep track of the IT assets constituting the current IT landscape, e.g. identifying IT assets, recording their properties and relationships to each other as well as updating these descriptions when the structure of the landscape changes. Second, these activities also include all the actions intended to assess the overall adequacy of the available IT assets (sometimes called 
as "health checking") and their fitness for the current and future business needs.

This activity area is typically supported by various EA artifacts accurately capturing the current state of the IT landscape. These EA artifacts may be titled as architectural repositories, asset registers, system inventories, application master lists or represent multiple separate graphical models, e.g. information exchange diagrams. Often this architectural information is stored in specialized EA repositories based on commercial software tools for EA.

All organizational benefits associated with this activity area can arguably be best summarized to the general rationalization and optimization of the IT landscape. This optimization implies several related aspects including lowered complexity and overhead, simplification of the landscape structure, increased reuse and decreased duplication of IT assets as well as enhanced agility in terms of planning new IT initiatives.

Essentially the only considerable blocker associated with the IT asset management activity area mentioned by the interviewees is the insufficient tool support making the tracking, maintenance and analysis of extensive architectural information rather problematic, time consuming and clumsy.

"The first year of enterprise architecture was about finding its fit in terms of the right tool to use. [...] We used [one tool] as our key architecture tool, but I would not say that it actually helped us greatly” (\#18)

\subsection{Opportunity assessment}

The opportunity assessment activity area encompasses all activities of architects related to evaluating possible options for addressing specific business needs with IT. These activities typically include clarifying the original business need and goals of the initiative, identifying available solution implementation options, assessing their architectural and technical feasibility, developing more detailed solution proposals, estimating their costs, timelines and risks and finally contributing to their business cases.

This activity area typically leverages various EA artifacts providing high-level views of proposed IT initiatives at different stages of their approval. These EA artifacts may include idea-stage project briefs with very abstract solution suggestions, early-stage options papers with the analysis of available solution alternatives, finalized conceptual architectures with rather elaborate descriptions of IT solutions and somewhat more technical preliminary solution architectures with necessary IT-specific details.
Organizational benefits resulting from this activity area are associated primarily with the ability to realize greater business value from respective IT investments. First, adequate opportunity assessment helps achieve better clarity and transparency of anticipated business benefits. Second, it allows estimating benefit-to-cost ratios and ensuring efficiency of IT investments. Third, it also helps mitigate possible risks associated with the implementation of corresponding IT solutions and improve the overall quality of project delivery.

Three different groups of blockers associated with the opportunity assessment activity area have been reported by the interviewees. First, in some cases architects focus excessively on the technical side of IT initiatives and pursue mostly the objectives related to IT and set by the CIO, instead of ensuring the achievement of business objectives. Second, in some public sector organizations the use of EA is mandated legislatively, but the genuine value of architectural involvement in initiatives is not recognized. Third, some business managers do not feel comfortable having negotiations with architects.

\subsection{Project governance}

The project governance activity area encompasses all activities of architects related to reviewing and approving the implementation plans for new IT projects. These activities often include studying proposed system designs as part of the project lifecycle, ensuring their compliance with the established implementation standards, discussing possible deviations, approving justified exceptions and giving respective dispensations.

This activity area is usually supported by a very broad variety of EA artifacts providing certain rules relevant to IT projects against which these projects can be assessed. Most often these EA artifacts include high-level policies and abstract principles, technical standards and detailed guidelines, recommended technologies and patterns (building blocks representing reusable solutions to typical problems) as well as conceptual data models.

Most prominent organizational benefits of this activity area are related to the ensuing budget economy, cost savings and lowered total cost of ownership (TCO) closely associated with the standardization of technologies and implementation approaches. These benefits may be realized through reducing the number of supported technologies, products and vendors as well as through achieving considerable economies of scale. Additionally, standardizing technologies and data structures also leads to improved technical and logical interoperability across the organizational IT landscape. 
The first clear blocker associated with the project governance activity area mentioned by the interviewees is the resistance to the restrictions imposed by architecture among project teams. The second and less common blocker related to this activity area is the lack of support for architecture governance from senior IT leadership (e.g. CIO) and the resulting inability of architects to enforce compliance with established standards.

\subsection{Communication and coordination}

The communication and coordination activity area encompasses all activities of architects related to the overall coordination of business and IT efforts in the organization. These activities typically include identifying key business and IT actors and decisionmakers, engaging with relevant stakeholders, establishing a productive dialog and trusted relationships with these stakeholders, understanding their genuine interests, concerns and plans and trying to influence these plans via informing them on other stakeholders' views and opinions.

Unlike all the previous activity areas described earlier, this area can be considered as "general" and is not associated closely with any particular types of EA artifacts. Although various EA artifacts can be certainly used as part of this activity area when they are necessary, this area relies more on verbal communication, than formal documents.

All organizational benefits of this activity area are associated with better partnership and closer collaboration between business and IT communities within the organization. Specifically, these benefits often include achieving more intensive communication and networking between various decision-makers, building trustful relationships and deepening mutual understanding between them, faster knowledge sharing, dissemination of ideas and coordination of plans.

Essentially the single major blocker associated with the communication and coordination activity area reported by the interviewees is poor communication skills of architects that prevent achieving mutual understanding with other EA stakeholders as well as the inability of architects to find an appropriate language for participating in direct conversations with these stakeholders, especially with senior business leaders.

\subsection{Consulting and mentoring}

The consulting and mentoring activity area encompasses all activities of architects focused on educating, advising and guiding other organizational actors. These activities may include mentoring project teams or less senior architects in various technical areas, educating business leaders regarding the opportunities and limitations of specific technologies, advising CIOs on the subjects relevant to the long-term IT strategy and consulting other IT managers on the questions related to the structure of the existing IT landscape and its capabilities.

Similarly to the communication and coordination activity area, this activity area arguably also cannot be associated directly with any particular types of EA artifacts. The primary "resource" in this activity area is unique expertise and deep knowledge possessed by architects, rather than EA artifacts themselves, though some EA artifacts can be leveraged if beneficial or necessary.

Key organizational benefits of this activity area are related primarily to achieving better overall consistency of IT-related planning decisions made in the organization. Adhering to the same line of thinking can lead to more holistic approach towards architectural planning and decision-making. Following the same course of action in similar situations and contexts contributes to harmonization of different aspects and elements of the organization.

The first significant blocker associated with the consulting and mentoring activity area mentioned by the interviewees is proving the value of architects' competence to the rest of the organization, or "finding a customer for the consulting service". The second blocker related to this activity area is the reputation and general perception of architecture as a blocker, rather than as enabler.

\subsection{Audit of mergers and acquisitions}

Finally, the audit of mergers and acquisitions activity area encompasses all activities of architects related to scrutinizing and analyzing the IT landscapes of other organizations prior to closing respective merger and acquisition deals. These activities are often accomplished by architects as part of more general due diligence procedures conducted before mergers and acquisitions of other companies.

Analogously to the two previous activity areas, this area is also largely artifacts-neutral in nature and can be hardly linked to specific types of EA artifacts. Instead, this activity area is driven predominantly by the intimate knowledge of the organizational IT landscape, its overall structure, constituting systems and underlying technologies possessed by architects, though various EA artifacts still can be used within this activity area when they are necessary.

All organizational benefits associated with this activity area can be related to a more adequate 
assessment of implications and consequences of mergers and acquisitions between different companies from the perspective of IT. For instance, active participation of architects in due diligence processes helps think ahead more proactively, estimate architectural compatibility between two landscapes, foresee potential problems and better plan necessary integration efforts in advance.

During our interviews with EA practitioners no articulate blockers that can be related specifically to the audit of mergers and acquisitions activity area have been identified.

\section{Discussion of findings}

The analysis of EA artifacts, activities, benefits and blockers reported by the interviewees suggests a number of interesting insights into EA practice.

\subsection{Diversity of activities}

The eight identified activity areas, as well as 11 underlying activities (see Figure 1), are very diverse in nature and range from organization-wide capability modeling to reviewing designs of separate IT systems. These observations support the view of an architect as a "jack of all trades" capable of communicating with business executives, mid-level managers and project teams and converting high-level business plans into low-level IT actions.

\subsection{EA artifacts and activities}

On the one hand, the eight identified activity areas suggest that specific types of EA artifacts are closely associated with certain activities that they support, but irrelevant to other activities. Moreover, no "generalpurpose" EA artifacts were mentioned by architects.

On the other hand, though EA practices are closely associated with EA artifacts [1, 2], not all activities mentioned by the interviewed architects can be related directly to any specific EA artifacts. This observation suggests that the work of an architect is not limited only to creating and using EA artifacts, but requires a much broader focus and also includes such essential activities as communication with various stakeholders, coordinating their activities, consulting business leaders, advising IT leaders and mentoring more junior IT specialists.

\subsection{Activity-specific nature of EA benefits and blockers}

While the existing EA literature tends to discuss the benefits and problems of "EA management" [11, 12], "EA programs" and "EA projects" [13, 14] or simply "enterprise architecture" [15, 16], our analysis shows that most reported benefits and blockers associated with EA practice can be actually related specifically to certain activity areas, rather than just to "enterprise architecture".

For example, clarity of priorities may result from business capability modeling but not from project governance, while reduced duplication may ensue from IT asset management but unlikely to follow from opportunity assessment. Similarly, the presence of cultural barriers between business and IT may be a significant blocker for business capability modeling but is irrelevant to IT asset management, while insufficient tool support may preclude effective IT asset management but it is of little or no relevance to opportunity assessment.

\subsection{Detailed view of EA practice}

While the existing literature often considers EA practice largely as a set of unspecified activities where some plans are produced and the alignment of business and IT is achieved [12, 13, 16], the eight specific activity areas identified in our study (see Figure 1) allow deconstructing the complex phenomenon of EA practice into a number of more definite discrete components. Each of these components essentially constitutes a separate subpart of EA practice embracing its own artifacts, participants, benefits and blockers. These activity areas can be taken as an initial basis for more detailed studies of EA practice by other researchers and thereby contribute to further development of the EA discipline.

\section{Conclusion}

This study provides an initial step necessary to explore EA practice in depth and "decompose" it into a number of more basic elements: artifacts, activities, benefits and blockers. The study identified eight distinct activity areas which capture an overall meaning of EA practice at a rather detailed level.

However, during the interviews the participating architects mentioned a very wide diversity of activities that they perform in their organizations. In order to manage this diversity and reduce it into a meaningful conceptual model, we focused primarily on artifacts, activities, blockers and benefits mentioned in an 
explicit form at least by 2-3 different architects. For this reason, a number of other activities mentioned only sporadically, briefly or implicitly have not been included into the resulting model. These missing activities include some "exotic" activities (e.g. managing vendor relationships and budgeting) as well as some narrow activities that can be considered as elements of more general activities (e.g. selection of technologies and architecture debt management). Therefore, full theoretical saturation on architects' activities might not have been achieved. This fact can be considered as a limitation of our study.

Nevertheless, we believe this study offers a sound contribution to EA research and practice. Each of the eight identified activity areas essentially represents a separate "story" within EA practice that deserves a more thorough investigation to better understand its internal details. Exploring these activity areas separately in greater detail can be considered as a potential direction for future EA research. This study also helps practitioners better understand potential benefits and blockers of EA practice. Such knowledge could be used to devise appropriate strategies for overcoming these inhibitors and facilitating the realization of desired benefits.

\section{Acknowledgement}

We would like to thank the Australian Research Council for funding this study (DP14010024).

\section{References}

[1] Kotusev, S., "Enterprise Architecture and Enterprise Architecture Artifacts: Questioning the Old Concept in Light of New Findings", Journal of Information technology, 34(2), 2019, pp. 102-128.

[2] Niemi, E., and Pekkola, S., "Using Enterprise Architecture Artefacts in an Organisation", Enterprise Information Systems, 11(3), 2017, pp. 313-338.

[3] Greefhorst, D., and Proper, E., Architecture Principles: The Cornerstones of Enterprise Architecture, Springer, Berlin, 2011.

[4] Ross, J.W., Weill, P., and Robertson, D.C., Enterprise Architecture as Strategy: Creating a Foundation for Business Execution, Harvard Business School Press, Boston, MA, 2006.

[5] Foorthuis, R., van Steenbergen, M., Brinkkemper, S., and Bruls, W.A., "A Theory Building Study of Enterprise Architecture Practices and Benefits", Information Systems Frontiers, 18(3), 2016, pp. 541-564.

[6] Pessi, K., Magoulas, T., and Hugoson, M.-A., "Enterprise Architecture Principles and Their Impact on the Management of IT Investments", Electronic Journal of Information Systems Evaluation, 14(1), 2011, pp. 53-62.
[7] Foorthuis, R.M., and Brinkkemper, S., "A Framework for Project Architecture in the Context of Enterprise Architecture", Proceedings of the 2nd Trends in Enterprise Architecture Research Workshop, 2007, pp. 51-60.

[8] Simon, D., Fischbach, K., and Schoder, D., "Enterprise Architecture Management and Its Role in Corporate Strategic Management", Information Systems and e-Business Management, 12(1), 2014, pp. 5-42.

[9] Makiya, G., "Integrating Enterprise Architecture and IT Portfolio Management Processes", Journal of Enterprise Architecture, 4(1), 2008, pp. 27-40.

[10] Armour, F.J., Emery, C., Houk, J., Kaisler, S.H., and Kirk, J.S., "The Integrated Enterprise Life Cycle: Enterprise Architecture, Investment Management, and System Development", in (Saha, P., 'ed.'): Handbook of Enterprise Systems Architecture in Practice, Information Science Reference, Hershey, PA, 2007, pp. 237-252.

[11] Schmidt, C., and Buxmann, P., "Outcomes and Success Factors of Enterprise IT Architecture Management: Empirical Insight from the International Financial Services Industry", European Journal of Information Systems, 20(2), 2011, pp. 168-185.

[12] Lohe, J., and Legner, C., "Overcoming Implementation Challenges in Enterprise Architecture Management: A Design Theory for Architecture-Driven IT Management (ADRIMA)", Information Systems and e-Business Management, 12(1), 2014, pp. 101-137.

[13] Levy, M., "'Shelfware' or Strategic Alignment? An Enquiry into the Design of Enterprise Architecture Programs", Proceedings of the 20th Americas Conference on Information Systems, 2014, pp. 1-12.

[14] Alaeddini, M., and Salekfard, S., "Investigating the Role of an Enterprise Architecture Project in the Business-IT Alignment in Iran", Information Systems Frontiers, 15(1), 2013, pp. 67-88.

[15] Bradley, R.V., Pratt, R.M., Byrd, T.A., and Simmons, L.L., "The Role of Enterprise Architecture in the Quest for IT Value", MIS Quarterly Executive, 10(2), 2011, pp. 73-80.

[16] Dang, D.D., and Pekkola, S., "Root Causes of Enterprise Architecture Problems in the Public Sector", Proceedings of the 20th Pacific Asia Conference on Information Systems, 2016, pp. 1-16.

[17] Kotusev, S., Singh, M., and Storey, I., "Investigating the Usage of Enterprise Architecture Artifacts", Proceedings of the 23rd European Conference on Information Systems, 2015, pp. 1-12.

[18] Boh, W.F., and Yellin, D., "Using Enterprise Architecture Standards in Managing Information Technology", Journal of Management Information Systems, 23(3), 2007, pp. 163-207.

[19] Khosroshahi, P.A., Hauder, M., Volkert, S., Matthes, F., and Gernegross, M., "Business Capability Maps: Current Practices and Use Cases for Enterprise Architecture Management", Proceedings of the 51st Hawaii International Conference on System Sciences, 2018, pp. 4603-4612. 
[20] Peels, F., Bons, R., and Plomp, M., "The Business Value of Enterprise Data Models", Proceedings of the 22nd Americas Conference on Information Systems, 2016, pp. 110 .

[21] EA on a Page, "Enterprise Architecture on a Page (v1.3)", SK Publishing, http://eaonapage.com, 2018,

[22] Keller, W., "Using Capability Models for Strategic Alignment", in (Simon, D., and Schmidt, C., 'eds.'): Business Architecture Management: Architecting the Business for Consistency and Alignment, Springer, Berlin, 2015, pp. 107122.

[23] Toppenberg, G., Henningsson, S., and Shanks, G., "How Cisco Systems Used Enterprise Architecture Capability to Sustain Acquisition-Based Growth", MIS Quarterly Executive, 14(4), 2015, pp. 151-168.

[24] Narman, P., Franke, U., Konig, J., Buschle, M., and Ekstedt, M., "Enterprise Architecture Availability Analysis Using Fault Trees and Stakeholder Interviews", Enterprise Information Systems, 8(1), 2014, pp. 1-25.

[25] Narman, P., Holm, H., Ekstedt, M., and Honeth, N., "Using Enterprise Architecture Analysis and Interview Data to Estimate Service Response Time", Journal of Strategic Information Systems, 22(1), 2012, pp. 70-85.

[26] Kotusev, S., "Conceptual Model of Enterprise Architecture Management", International Journal of Cooperative Information Systems, 26(3), 2017, pp. 1-36.

[27] Ahlemann, F., Stettiner, E., Messerschmidt, M., and Legner, C., ('eds.'), Strategic Enterprise Architecture Management: Challenges, Best Practices, and Future Developments, Springer, Berlin, 2012.

[28] Kotusev, S., The Practice of Enterprise Architecture: A Modern Approach to Business and IT Alignment, SK Publishing, Melbourne, Australia, 2018.

[29] Blomqvist, S., Halen, M., and Helenius, M., "Connecting Enterprise Architecture with Strategic Planning Processes: Case Study of a Large Nordic Finance Organization", Proceedings of the 17th IEEE Conference on Business Informatics, 2015, pp. 43-50.

[30] Petrikina, J., Drews, P., Schirmer, I., and Zimmermann, K., "Integrating Business Models and Enterprise Architecture", Proceedings of the 9th Trends in Enterprise Architecture Research Workshop, 2014, pp. 47-56.

[31] Barateiro, J., Antunes, G., and Borbinha, J., "Manage Risks Through the Enterprise Architecture", Proceedings of the 45th Hawaii International Conference on System Sciences, 2012, pp. 3297-3306.

[32] Aitken, C., "Enterprise Architecture, Strategic Management, and Information Management", in (Doucet, G., Gotze, J., Saha, P., and Bernard, S., 'eds.'): Coherency Management: Architecting the Enterprise for Alignment, Agility and Assurance, AuthorHouse, Bloomington, IN, 2009, pp. 121-155.

[33] Radeke, F., "Toward Understanding Enterprise Architecture Management's Role in Strategic Change: Antecedents, Processes, Outcomes", Proceedings of the 9th
International Conference on Wirtschaftsinformatik, 2011, pp. 497-507.

[34] Emery, C., Faison, S.M., Houk, J., and Kirk, J.S., "The Integrated Enterprise: Enterprise Architecture, Investment Process and System Development", Proceedings of the 40th Hawaii International Conference on System Sciences, 2007, pp. 1-11.

[35] Kotusev, S., "Enterprise Architecture: What Did We Study?", International Journal of Cooperative Information Systems, 26(4), 2017, pp. 1-84.

[36] Valorinta, M., "IT Alignment and the Boundaries of the IT Function", Journal of Information technology, 26(1), 2011, pp. 46-59.

[37] Bradley, R.V., Pratt, R.M., Byrd, T.A., Outlay, C.N., and Wynn Jr, D.E., "Enterprise Architecture, IT Effectiveness and the Mediating Role of IT Alignment in US Hospitals", Information Systems Journal, 22(2), 2012, pp. 97-127.

[38] Ajer, A.K., and Olsen, D.H., "Enterprise Architecture Challenges: A Case Study of Three Norwegian Public Sectors", Proceedings of the 26th European Conference on Information Systems, 2018, pp. 1-17.

[39] Seppanen, V., Heikkila, J., and Liimatainen, K., "Key Issues in EA-Implementation: Case Study of Two Finnish Government Agencies", Proceedings of the 11th IEEE Conference on Commerce and Enterprise Computing, 2009, pp. 114-120.

[40] Banaeianjahromi, N., and Smolander, K., "Understanding Obstacles in Enterprise Architecture Development", Proceedings of the 24th European Conference on Information Systems, 2016, pp. 1-15.

[41] Chuang, C.-H., and van Loggerenberg, J., "Challenges Facing Enterprise Architects: A South African Perspective", Proceedings of the 43rd Hawaii International Conference on System Sciences, 2010, pp. 1-10.

[42] Hauder, M., Roth, S., Matthes, F., and Schulz, C., "An Examination of Organizational Factors Influencing Enterprise Architecture Management Challenges", Proceedings of the 21st European Conference on Information Systems, 2013, pp. 1-12.

[43] Lucke, C., Krell, S., and Lechner, U., "Critical Issues in Enterprise Architecting - A Literature Review", Proceedings of the 16th Americas Conference on Information Systems, 2010, pp. 1-11.

[44] Creswell, J.W., Qualitative Inquiry and Research Design: Choosing Among Five Approaches, Sage, 2nd edn, Thousand Oaks, CA, 2007.

[45] Strauss, A.L., and Corbin, J.M., Basics of Qualitative Research: Techniques and Procedures for Developing Grounded Theory, Sage, 2nd edn, Thousand Oaks, CA, 1998. 